\title{
Avaliação dos resultados do tratamento cirúrgico de pacientes portadoras de endometriose do septo retovaginal
}

\author{
Evaluation of the results of surgical treatment of patients with \\ endometriosis of the rectovaginal septum
}

Walid Makin Fahmy ${ }^{1}$, Reginaldo Coelho Guedes Lopes ${ }^{2}$, José Francisco Dória Ramos ${ }^{3}$, Fausto Farah Baracat ${ }^{4}$, Ana Maria Pereira Gomes ${ }^{5}$, Gilberto Kendi Takeda ${ }^{6}$, Daniela Siqueira Prado ${ }^{7}$

\section{RESUMO}

Objetivo: avaliar os resultados dos 14 primeiros casos de tratamento cirúrgico videolaparoscópico de pacientes com endometriose profunda do septo retovaginal no Setor de Endoscopia Ginecológica do Hospital do Servidor Público Estadual "Francisco Morato de Oliveira". Métodos: foi realizada análise retrospectiva com dados retirados de prontuários, associada ao atendimento ambulatorial pós-operatório das 14 pacientes operadas entre fevereiro de 2002 e fevereiro de 2004 , apresentando as seguintes características: a idade das pacientes variou de 33 a 44 anos, com média de 38,4; a paridade variou de 0 a 3, com média de 1,1; os principais sintomas pré-operatórios foram: dismenorréia em 14 (100\%), dispareunia de profundidade em $12(85,7 \%)$, dor pélvica acíclica em 10 (71,4\%), dor à evacuação em duas (14,3\%), enterorragia em duas (14,3\%) e infertilidade em duas (14,3\%). A dosagem plasmática do CA-125 esteve entre 3,6 e 100,3 U/mL, com média de 52,9 U/mL. Resultados: o exame anatomopatológico das lesões do septo retovaginal foi compatível com endometriose em nove $(64,3 \%)$ pacientes. Quanto à sintomatologia dolorosa, houve regressão total em sete (50\%) pacientes, melhora de mais de $80 \%$ em duas (14,3\%), sem melhora em quatro $(28,6 \%)$ e piora em uma $(7,1 \%)$. A incidência de complicações foi de $14,3 \%$, sendo uma lesão de ureter associada a lesão no sigmóide e uma lesão retal diagnosticada no $8^{\circ}$ dia de pós-operatório. Conclusão: pode-se concluir que a endometriose profunda do septo retovaginal pode ser tratada pela cirurgia laparoscópica, com baixa morbidade, e trazendo alívio dos sintomas para a maioria das pacientes.

PALAVRAS-CHAVE: Endometriose/cirurgia; Doenças vaginais/cirurgia; Doenças retais/cirurgia; Resultados do tratamento

\section{ABSTRACT}

Purpose: to evaluate the results of 14 cases of laparoscopic surgical treatment of patients with deep endometriosis of the rectovaginal septum in the Sector of Gynecological Endoscopy of the 'Hospital do Servidor Público Estadual "Francisco Morato de Oliveira", Methods: a retrospective analysis was accomplished with data from the records, associated with postoperative evaluation of the patients operated between February 2002 and February 2004. The patients' age varied from 33 to 44 years, with a mean of 38.4. The parity ranged from 0 to 3 , with a mean of 1.1 . The main preoperative symptoms were: dysmenorrhea in $14(100 \%)$, deep dyspareunia in $12(85.7 \%)$, non-ciclic pelvic pain in $10(71.4 \%)$, pain at defecation in two (14.3\%), rectal bleeding in two (14.3\%), and infertility in two (14.3\%). The plasma level of CA-125 ranged from 3.6 to $100.3 \mathrm{U} /$ $\mathrm{mL}$, with a mean of $52.9 \mathrm{U} / \mathrm{mL}$. Results: the histological examination of the lesions of the rectovaginal septum was compatible with endometriosis in nine $(64.3 \%)$ patients. Concerning painful symptoms, there was total regression in seven (50\%) patients, partial regression (more than $80 \%$ relief) in two $(14.3 \%)$, no improvement in four $(28.6 \%)$, and worsening in one (7.1\%). The incidence of complications was 14.3\%: a ureter lesion associated with lesion of the sigmoid and a lesion of the rectum diagnosed on the 8th postoperative day. Conclusion: it can be concluded that endometriosis of the rectovaginal septum can be treated through laparoscopic surgery with low morbidity, leading to a complete or almost complete relief of the symptoms in most of the patients.

KEYWORDS: Endometriosis/surgery; Vaginal diseases/surgery; Retal diseases/surgery; Treatment outcome

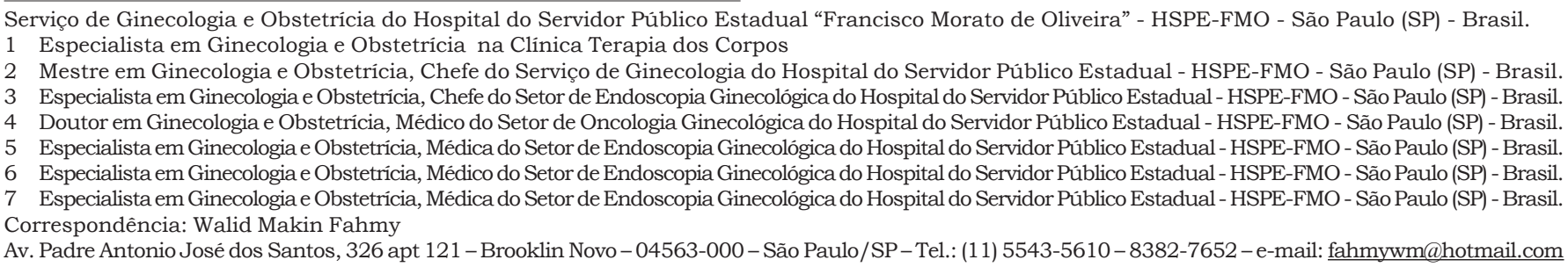




\section{Introdução}

A endometriose do septo retovaginal foi relatada por Sampson no início do século passado, que a descreveu como "um processo aderencial extenso na escavação reto-uterina, obliterando sua porção inferior e unindo a cérvix ou porções inferiores do corpo do útero ao reto, com invasão de tecido endometrial no colo do útero e no reto", sendo desta forma dificil obter-se a localização precisa da lesão ${ }^{1,2}$

A endometriose profunda do septo retovaginal é uma das formas mais graves desta doença. São lesões de formato esférico e localizadas profundamente na escavação reto-uterina. À laparoscopia são visualizadas somente como pequenas lesões típicas. Além disso, podem-se observar processos aderenciais entre o reto e o útero, assim como dilatação ureteral quando há comprometimento do paramétrio. As lesões são freqüentemente mais palpáveis do que visiveis e coexistem com dismenorréia progressiva, dispareunia, disquesia e dor pélvica baixa que se irradia posteriormente à região sacrococcígea, mas podem também ser assintomáticas ${ }^{3}$. Quando a parede intestinal é acometida, geralmente ocorre dor à evacuação, tenesmo, sensação de peso anorretal e sangramento retal cíclico ${ }^{4-6}$. O exame físico revela nódulos na escavação reto-uterina e nos ligamentos útero-sacrais. A terapia medicamentosa não possibilita resultados satisfatórios e nenhuma droga ou conjunto de drogas consegue erradicar a doença. Existe somente alivio temporário dos sintomas, mas as dores recorrem, comumente, após seis a 12 meses da interrupção da terapia, em $70 \%$ dos casos; pode, porém, ser boa opção para pacientes que não desejam engravidar ou que tenham os sintomas controlados com medicação ${ }^{6}$. Em pacientes assintomáticas, somente com endometriose do septo retovaginal, a conduta expectante também pode ser adotada, visto que a progressão da doença e o aparecimento de sintomas ocorrem em menos de dez por cento dos ca$\operatorname{sos}^{3}$. A excisão completa da lesão é hoje a melhor forma de tratamento nos casos em que a paciente tem desejo de engravidar ou é sintomática ${ }^{7}$. Com a cirurgia é possivel a análise histológica e a retirada das lesões profundas causadoras dos sintomas. O progresso da cirurgia laparoscópica nos últimos anos tem possibilitado às pacientes com endometriose profunda do septo retovaginal tratamento seguro, com rápida recuperação pós-operatória e baixa morbidade ${ }^{7,8}$. Este trabalho tem como objetivo avaliar os resultados obtidos em um grupo de pacientes submetidas a tratamento cirúrgico videolaparoscópico.
Pacientes e Métodos

Este estudo foi conduzido no Setor de Endoscopia Ginecológica do Serviço de Ginecologia e Obstetrícia do Hospital do Servidor Público Estadual "Francisco Morato de Oliveira", após aprovação pela Comissão de Ética em Pesquisa dessa instituição. Foi realizada análise retrospectiva, com dados retirados de prontuários, associada ao atendimento ambulatorial pós-operatório de todas as pacientes submetidas à laparoscopia para tratamento da endometriose profunda do septo retovaginal.

Foram incluídas no estudo 14 pacientes operadas entre fevereiro de 2002 e de 2004 . Não houve exclusão de nenhuma, pois todas apresentaram dados devidamente preenchidos no prontuário e foram acompanhadas após a cirurgia até a conclusão deste trabalho. O diagnóstico de endometriose profunda foi feito clinicamente pela sintomatologia (dismenorréia, dispareunia, dor pélvica, sangramento retal) e pelo exame físico, isto é, pelo achado ao toque vaginal e retal de nódulo e/ou endurecimento palpável na escavação reto-uterina, que esteve presente em 12 pacientes. Dentre as 14 pacientes, 11 (78,6\%) já haviam sido submetidas a pelo menos uma laparoscopia prévia (variando de uma a três cirurgias) e portanto já apresentavam diagnóstico cirúrgico anterior. Para complementar o diagnóstico utilizou-se a dosagem de CA-125 plasmático e ainda a ultra-sonografia transvaginal, a transretal e a retosigmóido/colonoscopia quando da suspeita de invasão da parede intestinal. A dosagem plasmática do CA-125 variou de 3,6 a 100,3 U/mL, com média de 52,9 U/mL. Foram realizadas cinco ultrasonografias transretais: uma foi normal e quatro revelaram nódulos no septo retovaginal, sendo que um deles envolvia a camada muscular do reto. A ultra-sonografia transvaginal demonstrou endometrioma de ovário em cinco pacientes e miomas intramurais em duas. Nas outras pacientes o exame foi normal. A colonoscopia foi feita em sete pacientes e não identificou nenhuma lesão suspeita de endometriose na mucosa retal, apenas abaulamentos e dobras em dois exames.

As pacientes foram submetidas a um preparo intestinal 48 horas antes da cirurgia. Onze $(78,6 \%)$ pacientes foram tratadas com análogo de GnRH, três meses antes da cirurgia. As pacientes foram amplamente informadas sobre os riscos da cirurgia e de possivel extensão para laparotomia, para colostomia ou para ressecção intestinal.

A técnica cirúrgica consistiu em separar a parede retal anterior da parede vaginal posterior, 
até atingir uma área de tecido sadio. Toda a área afetada pela doença foi então ressecada com tesoura e corrente elétrica monopolar com penetração na parede retal, a depender de cada caso. Quando a ressecção era restrita à serosa do reto, a área operada não era suturada, mas quando atingia a camada muscular ou a luz do reto, realizava-se a sutura em dois ou três planos. Se a lesão atingia a mucosa retal e tinha mais de $2 \mathrm{~cm}$ de extensão optava-se pela ressecção e anastomose términoterminal do reto com grampeadores, o que ocorreu em duas pacientes. Houve conversão para laparotomia em uma paciente com intuito de reparar uma lesão no reto e outra no ureter.

As pacientes foram avaliadas antes e após a cirurgia com seguimento que variou de seis meses a dois anos. A idade das pacientes variou entre 33 e 44 anos, com média de 38,4. Doze $(85,8 \%)$ eram brancas e duas $(14,3 \%)$ negras. A paridade oscilou de 0 a 3, com média de 1,1. A idade da menarca ocorreu entre 9 e 14 anos, com média de 12,8 . Doze $(85,8 \%)$ pacientes eram eumenorréicas. A doença associada mais encontrada foi a hipertensão arterial sistêmica, presente em três $(21,4 \%)$ pacientes. O exame físico revelou um nódulo na escavação reto-uterina em oito pacientes e um endurecimento da mesma em quatro. Duas não apresentaram alteração ao exame físico. Apenas uma apresentava lesão vesiculosa arroxeada na vagina. No que se refere à sintomatologia préoperatória (Tabela 1), dismenorréia foi o sintoma relatado por todas as pacientes $(100 \%)$, seguida de dispareunia de profundidade em 12 (85,7\%), dor pélvica acíclica em 10 (71,4\%), dor à evacuação em duas $(14,3 \%)$, enterorragia em duas $(14,3 \%)$ e infertilidade em duas (14,3\%). Foram também avaliados os achados cirúrgicos no que diz respeito às estruturas pélvicas e abdominais acometidas pela endometriose, a evolução da sintomatologia referida previamente pela paciente, o aparecimento de outros sintomas, o tipo de cirurgia realizada com suas complicações, além do exame anatomopatológico.

Tabela 1 - Sintomatologia relatada previamente à cirurgia.

\begin{tabular}{llc}
\hline Sintomas & $\mathbf{n}$ & $\%$ \\
\hline Dismenorréia & 14 & 100 \\
Dispareunia de profundidade & 12 & 85,8 \\
Dor pélvica acíclica & 10 & 71,5 \\
Dor à evacuação & 2 & 14,3 \\
Enterorragia & 2 & 14,3 \\
Infertilidade secundária & 2 & 14,3 \\
\hline $\mathrm{n}=$ número absoluto. & & \\
$\%=$ freqüência relativa percentual. & &
\end{tabular}

\section{Resultados}

O tempo médio entre o início dos sintomas e o primeiro diagnóstico cirúrgico foi de 13,6 meses. Os achados cirúrgicos relacionados à presença de lesões endometrióticas são mostrados na Tabela 2. Foi encontrada aderência de retossigmóide na escavação reto-uterina em todas as 14 pacientes; aderências anexiais na escavação retouterina em nove $(64,3 \%)$ pacientes; oito $(57,1 \%)$ apresentavam lesões no ligamento útero-sacro, uma $(7,1 \%)$ na bexiga e duas $(14,3 \%)$ na vagina. Em três casos $(21,4 \%)$, as lesões atingiram o intestino, com acometimento de camada muscular e mucosa, embora não visualizadas na colonoscopia; em quatro $(28,6 \%)$ as lesões eram ovarianas, uma $(7,1 \%)$ no colo uterino e uma $(7,1 \%)$ apresentava lesões tanto na goteira parietocólica bilateral quanto na cúpula diafragmática. Duas pacientes apresentavam mioma uterino.

Tabela 2 - Distribuição anatômica das lesões de endometriose $(n=14)$

\begin{tabular}{lccc}
\hline & Unilateral & Bilateral & Total $\mathbf{n}(\%)$ \\
\hline Útero-sacro & 2 & 6 & $8(57,1)$ \\
Bexiga & 1 & & $1(7,1)$ \\
Vagina & 2 & & $2(14,3)$ \\
Intestino & 3 & & $3(21,4)$ \\
Ovário & 1 & 3 & $4(28,6)$ \\
Colo & 1 & & $1(7,1)$ \\
Aderências anexiais na ERU & 5 & 4 & $9(64,3)$ \\
Aderência de retossigmóide na ERU & 14 & & $14(100)$ \\
Goteira parietocólica & & 1 & $1(7,1)$ \\
Cúpula diafragmática & 1 & & $1(7,1)$ \\
\hline
\end{tabular}

ERU = escavação reto-uterina.

$n=$ número absoluto.

$\%$ = freqüência relativa percentual.

Diante destes achados cirúrgicos foram tomadas as condutas apresentadas na Tabela 3. Foi realizada a dissecção do espaço retovaginal em todas as pacientes, com procedimentos acessórios dependendo do caso. Houve ressecção de nódulo do septo retovaginal em $12(85,7 \%)$ pacientes, ressecção de fibrose do septo em duas (14,3\%) pacientes, anexectomia unilateral em duas $(14,3 \%)$ e bilateral em duas (14,3\%), exérese de cápsula com drenagem de endometrioma unilateral em uma $(7,1 \%)$ e bilateral em duas $(14,3 \%)$, histerectomia em cinco $(37,5 \%)$, sendo duas subtotais e três totais, retossigmoidectomia em duas (14,3\%), ressecção de nódulo no ligamento útero-sacro em duas $(14,3 \%)$, ressecção de implantes na vagina em duas $(14,3 \%)$, ressecção de implantes em 
serosa de bexiga em uma $(7,1 \%)$ e miomectomia em duas $(14,3 \%)$. Importante lembrar que na mesma paciente houve diferentes lesões e diferentes procedimentos cirúrgicos.

Tabela 3 - Procedimentos cirúrgicos realizados para remoção das lesões endometrióticas.

\begin{tabular}{lr}
\hline Procedimentos cirúrgicos & $\mathbf{n}$ \\
\hline Dissecção do septo retovaginal & 14 \\
Ressecção de nódulo do septovaginal & 12 \\
Ressecção de fibrose septo retovaginal & 2 \\
Anexectomia & 2 \\
$\quad$ Unilateral & 2 \\
$\quad$ Bilateral & \\
Exérese de cápsula de endometrioma & 1 \\
$\quad$ Unilateral & 2 \\
$\quad$ Bilateral & 2 \\
Histerectomia subtotal & 3 \\
Histerectomia total & 2 \\
Retossigmoidectomia & 2 \\
Exérese de nódulo útero-sacro bilateral & 2 \\
Ressecção de implantes na vagina & 1 \\
Ressecção de implantes na serosa da bexiga &
\end{tabular}

Pacientes foram submetidas a mais de um procedimento.

O exame anatomopatológico das lesões no septo retovaginal foi compativel com endometriose em nove $(64,3 \%)$ pacientes, com adenomiose em uma $(7,1 \%)$, tecido fibromuscular em três $(21,4 \%)$ e, em uma $(7,1 \%)$, o septo não foi avaliado histologicamente.

No seguimento pós-operatório, no que diz respeito à sintomatologia dolorosa observamos: regressão total em sete $(50 \%)$ pacientes, melhora de mais de $80 \%$ em duas $(14,3 \%)$, sem melhora em quatro $(28,6 \%)$ e piora em uma $(7,1 \%)$. Alguns sintomas surgiram após a cirurgia, como dificuldade à evacuação em uma $(7,1 \%)$ e dor retal em uma $(7,1 \%)$; uma paciente $(7,1 \%)$ permaneceu com a mesma queixa de enterorragia prévia à cirurgia.

Ocorreram duas (14,3\%) complicações. Houve uma lesão de ureter esquerdo, que foi suturado e cateterizado com cateter duplo J, associada a lesão no sigmóide, que foi suturada após conversão para laparotomia. Outra complicação foi uma lesão de reto diagnosticada tardiamente no oitavo dia pós-operatório. A paciente foi tratada por laparotomia e colostomia transversa, com reconstrução do trânsito intestinal após cinco meses. Ambas as pacientes estão sendo acompanhadas e apresentam adequada função intestinal e urinária, não apresentando neste momento nenhuma alteração causada pela cirurgia.

\section{Discussão}

A cirurgia laparoscópica para ressecção da endometriose profunda do septo retovaginal é procedimento complexo que requer certo grau de experiência do cirurgião, porque são necessárias dissecções extensas próximas ao ureter, aos vasos uterinos e ao reto ${ }^{8}$. E por esta razão que estas pacientes são submetidas, quase sempre, a grande número de intervenções cirúrgicas não resolutivas $^{7}$. Em uma série de 169 pacientes com endometriose retrocervical, observou-se que $48 \%$ já haviam sido submetidas a duas ou mais cirurgias laparoscópicas ${ }^{5}$. Da mesma forma, entre 77 pacientes com endometriose colo-retal, 52\% haviam se submetido a tratamento cirúrgico prévio para endometriose ${ }^{9}$. Nas pacientes deste estudo, somente três $(21,4 \%)$ não haviam sido submetidas a nenhuma laparoscopia prévia, demonstrando, assim, a dificuldade no tratamento cirúrgico completo em uma única etapa, sendo em geral a primeira cirurgia apenas diagnóstica.

Para programar melhor a cirurgia, um aspecto a ser avaliado é o diagnóstico do envolvimento intestinal. Os sintomas que levantam as suspeitas são a obstipação alternada com diarréia, sangramento ou dor retal, dismenorréia, tenesmo e dispareunia ${ }^{9}$. Esses sintomas são causados por inflamação e fibrose associadas à endometriose profunda, causando uma reação esclerosante da parede intestinal que pode diminuir sua luz. Por esta razão os sintomas podem persistir mesmo que a endometriose não esteja ativa. O hipoestrogenismo devido à ooforectomia ou pela menopausa fisiológica não necessariamente faz regredir uma lesão estável ou uma reação de esclerose associada com lesões ativas e inativas ${ }^{9}$. O envolvimento intestinal deve ser suspeitado em toda paciente com endometriose grave (classificação de Acosta, 1973). Estima-se que metade das pacientes com endometriose grave têm algum grau de endometriose intestinal. Desta forma, em toda paciente com endometriose grave deve ser realizado preparo intestinal para que a cirurgia definitiva possa ser feita na primeira laparoscopia ${ }^{9}$. Em nossa casuística havia acometimento da camada muscular e da mucosa intestinal em três pacientes $(21,4 \%)$. Em duas foram realizadas ressecções segmentares intestinais, com resolução dos sintomas. Em uma paciente não foi feita cirurgia intestinal e ela permaneceu com sintomatologia de enterorragia, confirmando assim que a eficiência do tratamento depende da exérese completa da lesão. Analisando estudos referentes à ressecção de endometriose intesti- 
nal, foi realizada dissecção retal ou excisão por laparoscopia/laparotomia de endometriose coloretal em 169 pacientes. Em 35 meses de seguimento, 36\% das pacientes permaneceram com queixa de dor ${ }^{7}$. Entre 77 pacientes com endometriose colo-retal profunda tratadas com ressecção total da lesão (por ressecção em disco ou segmentar com anastomose término-terminal), com seguimento de um a nove anos, observou-se que $49 \%$ das pacientes tiveram alívio completo dos sintomas, 39\% melhora satisfatória, 11\% permanência dos sintomas e $1 \%$ piora dos mesmos. Já na ressecção colo-retal em 40 pacientes com endometriose intestinal, houve melhora estatisticamente significante da dor pélvica, dismenorréia, dispareunia e dor à evacuação ${ }^{10}$.

Assim como outros na literatura ${ }^{2}$, este trabalho sugere que as lesões profundas são geralmente multifocais, podendo envolver, simultaneamente, os ovários, os ligamentos útero-sacrais, a vagina, o intestino e a bexiga, provocando aderências anexiais e da escavação reto-uterina em grande número de pacientes (Tabela 2). Sendo assim, a eficiência do tratamento cirúrgico depende da radicalidade da exérese das lesões. Portanto, vários procedimentos intra-operatórios devem ser associados para que se tenha amplo sucesso no tratamento ${ }^{11}$, com conseqüente melhora da sintomatologia dolorosa. As pacientes que removeram completamente todo tecido ovariano tiveram melhor alivio dos sintomas ${ }^{9,12}$, o que foi observado ao avaliar 138 mulheres submetidas à histerectomia com diagnóstico de endometriose. Das pacientes nas quais foram preservados os ovários, $62 \%$ tiveram recorrência da dor, contra $10 \%$ nas quais os ovários foram removidos. A conservação ovariana foi associada com um risco relativo de recorrência da dor ${ }^{1,6,12}$. Em nosso estudo a ooforectomia bilateral foi realizada em duas pacientes, e destas uma apresentou regressão total da sintomatologia dolorosa no seguimento de um ano e a outra melhora de mais de $80 \%$ da sintomatologia dolorosa. Outros estudos têm relatado melhora significante da sintomatologia dolorosa, sem comparar os resultados com a retirada ou não do ovário: foram tratadas por videolaparoscopia e laser de $\mathrm{CO}_{2}, 185$ pacientes com endometriose do sigmóide, reto e do ligamento útero-sacro. Após a cirurgia, 83\% das pacientes apresentaram redução da dor, de moderada a completa, e o restante queixou-se de persistência ou até de piora da dor ${ }^{13}$. Em 26 pacientes com endometriose da escavação reto-uterina tratadas com cirurgia laparoscópica excisional, 88\% referiram dor discreta ou ausente após seis meses ${ }^{14}$.

Esses mesmos autores afirmam que a cirurgia melhora a sintomatologia dolorosa entre 64 e $88 \%$ e que a ressecção ovariana bilateral proporciona melhores resultados. Nosso trabalho mostrou melhora da sintomatologia dolorosa em $64,3 \%$ das pacientes. Em nosso serviço não é rotina a salpingooforectomia bilateral em pacientes abaixo dos 50 anos e, portanto, esta operação só foi realizada em casos de extenso comprometimento do tecido ovariano pela doença. A permanência dos sintomas pode ter sido relacionada à preservação ovariana, mas também à não-radicalidade da cirurgia, visto que as lesões são, em alguns casos, tecnicamente de difícil reconhecimento.

O diagnóstico e o tratamento da endometriose profunda são baseados no aspecto macroscópico da lesão. Entretanto, em algumas pacientes a histologia difere do aspecto macroscópico porque o implante contém apenas tecido denso fibrótico, ao invés de glândula ou de estroma endometrial, assim como em 209 pacientes com endometriose profunda, foi encontrado confirmação histológica em 142 (67,9\%) e, em 47 (22,5\%), apenas fibrose ${ }^{15}$. Da mesma forma, ao avaliarem 26 pacientes com endometriose da escavação reto-uterina, a endometriose foi confirmada histologicamente em 20 (77\%) pacientes, enquanto em seis $(23 \%)$ havia apenas tecido fibrótico ${ }^{14}$.

Em se tratando somente de endometriose intestinal, foi obtida confirmação anatomopatológica de endometriose em todas as 77 (100\%) pacientes operadas ${ }^{9}$. Outra abordagem histológica foi referida por Donnez et al. ${ }^{16}$ ao avaliarem 231 casos de endometriose retrocervical. A histologia demonstrou, em todas, nódulo similar ao adenomioma. Segundo os autores, era um nódulo circunscrito agregado a um tecido muscular liso e glândulas endometriais, sugerindo doença específica que se originaria de restos müllerianos presentes no septo retovaginal, e a denominaram adenomiose do septo retovaginal.

Neste trabalho obteve-se confirmação histopatológica de endometriose em nove pacientes $(64,0 \%)$, incluindo as duas que foram submetidas à ressecção intestinal.

A modificação das relações anatômicas conseqüentes à infiltração retroperitoneal das lesões faz com que a ressecção da endometriose profunda constitua fator de risco para complicações durante a cirurgia laparoscópica ${ }^{8}$. Além disso, a remoção cirúrgica da endometriose colo-retal apresenta complicações maiores, dependendo da extensão e profundidade da lesão retal ${ }^{10}$. Assim, em 169 casos de endometriose retrocervical, observaram-se lesões do trato urinário, intestinal e de vasos sangüíneos em nove $(5,4 \%)^{7}$, e em 77 pacientes com endometriose colo-retal, houve duas complicações $(2,6 \%)$, uma paciente com íleo para- 
lítico persistente e outra com aderências que causaram obstrução intestinal ${ }^{9}$. Ao tratar 185 pacientes com endometriose de sigmóide, reto e útero-sacro, houve perfuração intestinal como complicação em nove $(4,9 \%)^{13}$. Na ressecção colo-retal de 40 pacientes com endometriose intestinal, houve $10 \%$ de complicações, sendo três casos de fistula retovaginal e um de abscesso pélvico. Ocorreram também sete casos $(17,5 \%)$ de disfunção urinária transitória ${ }^{10}$.

Em nossa casuística observamos taxa de $12,4 \%$ de complicações, que é superior a apresentada na literatura. Esta diferença pode ser explicada pelo fato de terem sido os primeiros casos operados no Serviço de Ginecologia e Obstetrícia. Porém, ambas complicações puderam ser reparadas adequadamente, sem prejuízo na qualidade de vida a longo prazo.

Pode-se concluir que, apesar da agressividade da endometriose profunda do septo retovaginal, ela pode ser tratada satisfatoriamente pela cirurgia laparoscópica, com baixa morbidade, trazendo alívio dos sintomas na maioria das pacientes, apesar do pequeno número de casos analisados.

\section{Referências}

1. Abrão MS, Podgae CS, Ribeiro SC. Tratamento cirúrgico na endometriose: quando e como fazer. In: Abrão MS, editor. Endometriose: uma visão contemporânea. Rio de Janeiro: Revinter; 2000. p. 137-47.

2. Fauconnier A, Chapron C, Dubuisson JB, Vieira M, Dousset B, Breart G. Relation between pain symptoms and the anatomic location of deep infiltrating endometriosis. Fertil Steril. 2002;78(4):719-26.

3. Fedele L, Bianchi S, Zanconato G, Raffaelli R, Berlanda N. Is rectovaginal endometriosis a progressive disease? Am J Obstet Gynecol. 2004;191(5):1539-42.

4. Anaf V, Simon P, El Nakadi I, Simonart T, Noel JC, Buxant F. Impact of surgical resection of rectovaginal pouch of Douglas endometriotic nodules on pelvic pain and some elements of patients' sex life. J Am Assoc Gynecol Laparosc. 2001;8(1):55-60.
5. Anaf V, Simon P, El Nakadi I, Fayt I, Buxant F, Simonart T, et al. Relationship between endometriotic foci and nerves in rectovaginal endometriotic nodules. Hum Reprod. 2000;15(8):1744-50.

6. Emmanuel KR, Davis C. Outcomes and treatment options in rectovaginal endometriosis. Curr Opin Obstet Gynecol. 2005;17(4):399-402.

7. Varol N, Maher P, Healey M, Woods R, Wood C, Hill $D$, et al. Rectal surgery for endometriosis: shoud we be aggressive? J Am Assoc Gynecol Laparosc. 2003;10(2):182-9.

8. Chapron C, Dubuisson JB. Laparoscopic treatment of deep endometriosis located on the uterosacral ligaments. Hum Reprod. 1996;11(4):868-73.

9. Coronado C, Franklin RR, Lotze EC, Bailey HR, Valdes CT. Surgical treatment of symptomatic colorectal endometriosis. Fertil Steril. 1990;53(3):411-6.

10.Darai E, Thomassin I, Barranger E, Detchev R, Cortez A, Houry S, et al. Feasibility and clinical outcome of laparoscopic colorectal resection for endometriosis. Am J Obstet Gynecol. 2005; 192(2):394-400.

11. Chapron C, Fauconier A, Vieira M, Barakat H, Dousset B, Pansini V, et al. Anatomical distribution of deeply infiltrating endometriosis: surgical implications and proposition for a classification. Hum Reprod. 2003;18(1):157-61.

12.Namnoum AB, Hickman TN, Goodman SB, Gehlbach DL, Rock JA. Incidence of symptom recurrence after hysterectomy for endometriosis. Fertil Steril. 1995;64(5):898-902.

13.Nezhat C, Nezhat F, Pennington E. Laparoscopic treatment of infiltrative rectosigmoid colon and rectovaginal septum endometriosis by the technique of videolaparoscopy and the $\mathrm{CO}_{2}$ laser. $\mathrm{Br} \mathrm{J}$ Obstet Gynaecol. 1992;99(8):664-7.

14. Wood C, Maher P, Hill D. Laparoscopic removal of endometriosis in the pouch of Douglas. Aust N Z J Obstet Gynaecol. 1993;33(3):295-9.

15.Chapron C, Fauconnier A, Dubuisson JB, Barakat H, Vieira M, Breart G. Deep infiltrating endometriosis: relation between severity of dysmenorrhoea and extent of disease. Hum Reprod. 2003;18(4):760-6.

16.Donnez J, Nisolle M, Casanas-Roux F, Bassil S, Anaf V. Rectovaginal septum, endometriosis or adenomyosis: laparoscopic management in a series of 231 patients. Hum Reprod. 1995;10(3):630-5. 